The Israeli Journal of Aquaculture - Bamidgeh, IJA_69.2017.1411, 7 pages



The IJA appears exclusively as a peer-reviewed on-line open-access journal at http://www.siamb.org.il. To read papers free of charge, please register online at registration form.

Sale of IJA papers is strictly forbidden.

\title{
Isolation and Characterization of Acinetobacter Iwoffii from the Intestine of Grass Carp (Ctenopharyngodon idella)
}

\author{
Aijun Lü ${ }^{1,2 *}$, Zhen Wei ${ }^{1,2}$, Xiucai Hu ${ }^{2}$, Jingfeng Sun ${ }^{2}$, Chao Pei ${ }^{1}$, \\ Chao Zhang ${ }^{1}$, $\mathrm{Li} \mathrm{Li}^{1}$, AiLing $\mathrm{Li}^{3}$ \\ ${ }^{1}$ College of Fisheries, Henan Normal University, Xinxiang, 453007, China \\ 2 Tianjin Key Lab of Aqua-Ecology and Aquaculture, College of \\ Fisheries, Tianjin Agricultural University, Tianjin, 300384, China \\ ${ }^{3}$ College of Life Science and Food Engineering, Shaanxi Xueqian Normal \\ University, Xi'an 710061, China
}

Keywords: grass carp; Acinetobacter Iwoffii; isolation; identification; pathogenicity

\begin{abstract}
Acinetobacter Iwoffii infections are largely concentrated in mammals; very few reports are available for such infections in fish. In this study, a Gram-negative rod bacillus was isolated from the intestine of grass carp (Ctenopharyngodon idella), and designed as strain CY1. The isolated strain was then identified as $A$. Iwoffii according to morphological, physiological, and biochemical properties, and 16S rDNA sequencing. The phylogenetic tree constructed on the basis of $16 \mathrm{~S}$ rDNA sequences indicated that strain CY1 is most closely related to $A$. Iwoffii, and has $98.08 \%$ similarity with type strain ATCC17925. Experimental infection assays were conducted, and a cumulative mortality rate of $75 \%$ was observed in grass carp. Antimicrobial susceptibility test showed that the isolated strain CY1 was highly susceptible to most antimicrobial agents tested, including aminoglycosides, $\beta$-lactams, and quinolones. To our knowledge, this is the first report on pathogenic $A$. Iwoffii in grass carp.
\end{abstract}

\footnotetext{
*Corresponding author. Fax: +86-373-3328867, e-mail: lajand@126.com
} 


\section{Introduction}

Acinetobacter Iwoffii is an aerobic, non-fermentative gram-negative bacill (Rathinavelu et al., 2003; Takehiko et al., 2005; Ku et al., 2000), which is non-fastidious and can utilize a wide variety of substrates as their sole energy source (Rathinavelu et al., 2003). Hence, these organisms are generally ubiquitous and are frequently found in soil, water, dry environments, and hospitals (Rathinavelu et al., 2003). Due to its ubiquitous nature, it is a potential opportunistic pathogen in patients with impaired immune systems and it has been identified as a cause of nosocomial infections (Bergogne-Berezin et al., 1996; Forster et al., 1998). It is a well-known pathogen associated with gastritis, septicemia, pneumonia, meningitis, and urinary tract infections in humans (Luciano et al., 2007; Wareham et al., 2008; Sule et al., 1997; Idzenga et al., 2006; Medina et al., 2007; Kim et al., 2014). Infections caused by $A$. Iwoffii in previously reported cases have mostly concentrated in humans; few reports are available in fish (Kozińska et al., 2014; Dadar et al., 2016).

Grass carp (Ctenopharyngodon idella) is a long slender member of the minnow family native to large rivers in Asia. It is one of the most abundant freshwater fish in China, with production of over 3 million tons per year, which is $20 \%$ of the total yield of freshwater fish. However, bacterial disease outbreaks have caused great economic losses in cultured grass carp (Rathinavelu al et al., 2003). Some bacterial pathogens such as Shigella sp, Escherichia coli, and Serratia fonticola, have been isolated from diseased grass carp (Toyoshima et al., 2010), but there have been no reports about A. Iwoffii isolated from diseased grass carp.

In this study, a Gram-negative bacterium was isolated from the intestine of grass carp, and then identified as $A$. Iwoffii by $16 \mathrm{~S}$ rDNA gene sequences. The pathogenicity was confirmed in fish by challenge infection. The grass carp infected by $A$. Iwoffii also showed serious hemorrhages in pectoral, ventral, and anal fins. The first description of isolation and characterization of $A$. Iwoffii from grass carp provides a scientific reference for fish disease diagnostics.

\section{Materials and methods}

Isolation and culture of the bacterial pathogen from grass carp. Bacterial pathogens were isolated from intestinal samples of seemingly healthy grass carp according to a previous method (Hu et al., 2010). The grass carp were collected from a commercial fish farm at Xuzhou City, in China. Samples were streaked onto Luria-Bertani (LB) agar plates supplemented with tryptone $(10 \mathrm{~g} / \mathrm{L})$, yeast extract $(5 \mathrm{~g} / \mathrm{L})$, and $\mathrm{NaCl}(5 \mathrm{~g} / \mathrm{L})$ incubated at $28^{\circ} \mathrm{C}$ for $18-24 \mathrm{~h}$. Single colonies from plates were re-streaked on the same media to obtain pure isolates. The isolates were stored at $-80^{\circ} \mathrm{C}$ in LB broth at a final concentration of $15 \%$ glycerol.

Physiological and biochemical test. Morphological investigation was conducted using the gram-staining method. Biochemical tests were performed using a microbacteria biochemical test system (Tianhe Microbial Reagent Co., Ltd, China), including lysine decarboxylase, lysine decarboxylase, oxidase, arginine dihydrolase, and urease; arginine dihydrolase as well as urease; nitrate reductase, gluconate and DNase; reactions for Methyl red and Voges-Proskauer; acid production from sorbitol and erythritol, lactose, arabinose, raffinose, sorbose, etc. The micro-bacteria biochemical test tube was incubated in an incubator at $28^{\circ} \mathrm{C}$ for $48 \mathrm{~h}$.

Antimicrobial susceptibility test. The antimicrobial susceptibility tests of the isolate were conducted using Kirby-Bauer disc diffusion method on Mueller-Hinton agar plates, with results interpreted according to the guidelines of the Clinical and Laboratory Standards Institute. Each value is a mean of three replicates. The antimicrobial agents (Tianhe Microbial Reagent Co., Ltd, China) included were [The number in brackets indicates the dose of each antibiotic ( $\mu \mathrm{g}$ per disc)]: ampicillin (10 units), amoxicillin (10), carbenicillin (100), meropenem (10), imipenem (10), cefamandole (30), cefixime (5), cefotaxime (30), cephalothin (30), cephalexin (30), cefoperazone (75), piperacillin (100), amikacin (30), gentamicin (10), kanamycin (30), netilmicin (30), neomycin (30), streptomycin (10), tetracycline (30), chloramphenicol (30), nitrofurantoin (300), norfloxacin (10), ofloxacin(5),pefloxacin(10), enrofloxacin(5), enoxacin(10), 
sulfamethoxazole/trimethoprim (23.75/1.25), sulphafurazole (300), trimethoprim (5), azithromycin (15), erythromycin (15), teicoplanin (30), vancomycin (30), rifampicin (5), clindamycin (2), lincomycin (2).

$16 S$ rDNA gene sequences analysis. Total genomic DNA of the isolate strains was extracted with a column genomic DNA extraction kit according to the manufacturer's protocol. Oligonucleotide primers were used to amplify a 16S rDNA amplicon of approximately $1,500 \mathrm{bp}$. The primer sequences were generated according to the method of Vaneechoutte et al (Lü et al., 2016), and were as follows: 5'- AGAGTTTGATGGCTCAG 3' (forward primer) and 5'- AAGTCGTAACAAGGTAACCGTA -3' (reverse primer). PCR amplification conditions were as following: an initial denaturation step at $95^{\circ} \mathrm{C}$ for $2 \mathrm{~min}$; 30 cycles of $95^{\circ} \mathrm{C}$ for $30 \mathrm{~s}$, annealing for $30 \mathrm{~s}$ and $72^{\circ} \mathrm{C}$ for $1 \mathrm{~min}$; a final extension step at $72^{\circ} \mathrm{C}$ for $10 \mathrm{~min}$. PCR amplification was carried out in $25 \mu \mathrm{L}$ volumes of incubation buffer containing $1 \mu \mathrm{L}$ of bacterium solution, $1 \mu \mathrm{L} \times 2$ of $27 \mathrm{~F} / 1492 \mathrm{R}, 12.5 \mu \mathrm{L}$ of Master Mix, 9.5 $\mu \mathrm{L}$ of $\mathrm{ddH}_{2} \mathrm{O}$. The PCR product was analyzed by gel electrophoresis.

Experimental infections. Sixteen healthy grass carp were fed with commercial feed (Tongwei Co., Ltd, China) for the duration of the seven day infection experiment. The grass carp were divided into two groups with eight grass carp in each group. Fish were infected by intraperitoneal (i.p.) injection with $0.2 \mathrm{ml}$ dosage of approximately $3.83 \times 10^{8}$ $\mathrm{CFU} / \mathrm{ml}$. The control group was treated with $0.2 \mathrm{~mL}$ of $0.65 \%$ normal saline. The temperature of the water was about $20^{\circ} \mathrm{C}$ throughout this test.

\section{Results}

Morphology and biochemical characteristics of CY1 strain. One bacteria strain was isolated from grass carp intestines and tentatively named strain $\mathrm{CY} 1$. Through isolation and purification, the colonies of $\mathrm{CY} 1$ on LB plates looked circular, semitransparent, smooth, humid, and ridgy as shown in Fig. $1(\mathrm{~A})$. The bacterial cells were Gram-negative rod-shaped as shown in Fig. 1 (B). Biochemical tests results are summarized in Table 1, the results showed that $\mathrm{CY} 1$ was positive for xylose, fructose, xylitol, while was negative for gelatin, power, oxidase, etc. CY1 is weakly positive for arginine dihydrolase.

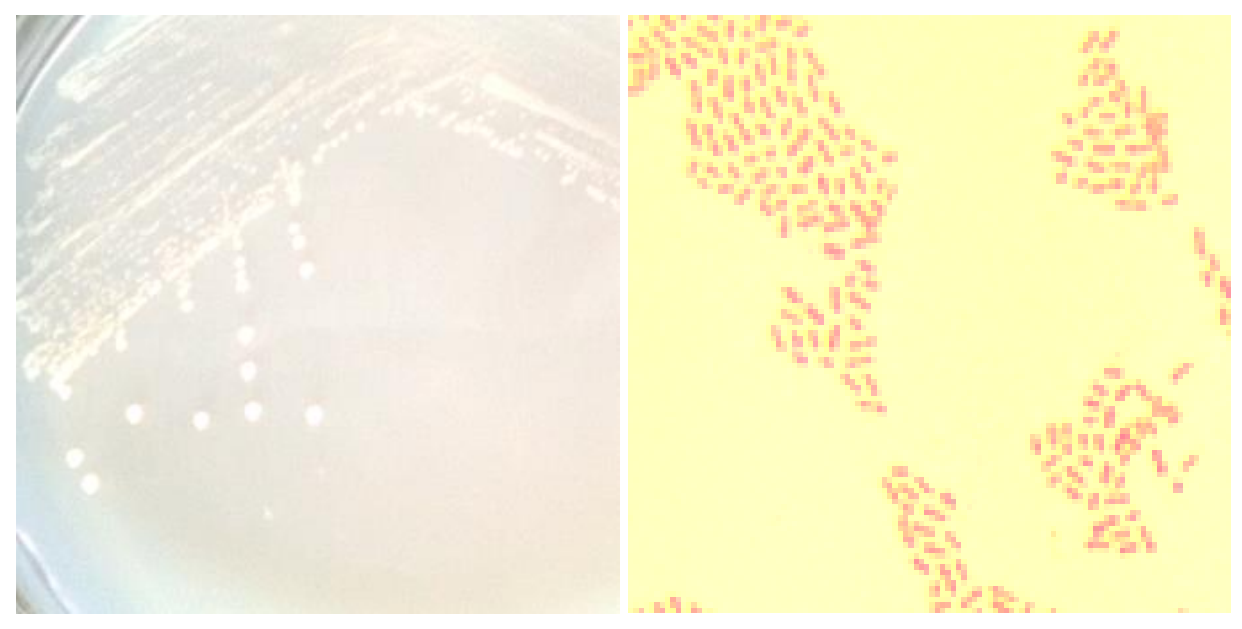

Fig. 1. A) The colonies of CY1 on LB plates looked circular, semitransparent, smooth, humid and ridgy. B) A. Iwoffii form Gram-negative rod-shaped clusters. 
Table 1. Morphological and biochemical properties of the strain CY1 isolated from grass carp.

\begin{tabular}{lclc}
\hline Characteristics & CY1 & Characteristics & CY1 \\
\hline Fructose & + & Glucose (gas) & - \\
Motility & - & Xylitol & + \\
Melibiose & - & Sorbitol & - \\
Oxidase & - & Raffinose & - \\
Lactose & - & Xylose & + \\
Arabinose & - & Mannose & - \\
Ornithine decarboxylase & - & Phenylalanine & - \\
Sucrose & - & deaminase & - \\
Lysine decarboxylase & - & Maltose & - \\
Glucose & - & Erythritol reductase & - \\
Arginine dihydrolase & \pm & Mannitol & - \\
Urease & - & Adonital & - \\
$\mathrm{N}-$ acetylglucosamine & - & Inositol & - \\
Nitrate reduction & - & MR & - \\
$\mathrm{H}_{2} \mathrm{~S}$ & - & Gluconate & - \\
VP & - & Citrate & - \\
\hline
\end{tabular}

Note: + : positive. - : negative. \pm : weak reaction.

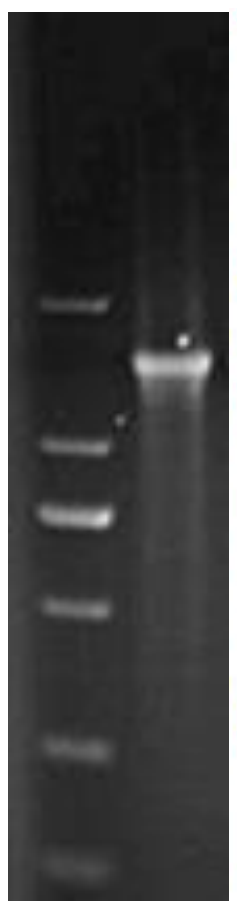

AGAGTTTGATCATGGCTCAGATTGAACGCTGGCGGCAGGCTTAACACATGCAAGTCGA GCGGGGAAAAGTAGCTTGCTACTTAACTTAGCGGCGGACGGGTGAGTAATACTTAGGA ATCTACCTAATAGTGGGGGACAACATCTCGAAAGGGATGCTAATACCGCATACGCCCTA CGGGGGAAAGCAGGGGACTCTTCGGAGCCTTGCGCTAATAGATGAGCCTAAGTCGGAT TAGCTAGTTGGTGGGGTAAAGGCCTACCAAGGCGACGATCT GTAGCGGGTCTGAGAGG ATGATCCGCCACACTGGGACTGAGACACGGCCCAGACTCCTACGGGAGGCAGCAGTG GGGAATATTGGACAATGGGGGGAACCCTGATCCAGCCATGCCGCGTGTGTGAAGAAGG CCTTTTGGTTGTAAAGCACTTTAAGCGAGGAGGAGGCTACTCTAGTTAATACCTAGAGA TAGTGGACGTTACTCGCAGAATAAGCACCGGCTAACTCTGTGCCAGCAGCCGCGGTAA TACAGAGGGTGCAAGCGTTAATCGGATTTACTGGGCGTAAAGCGTGCGTAGGTGGCCA ATTAAGTCAAATGTGAAATCCCCGAGCTTAACTTGGGAATTGCATTCGATACTGGTTGG CTAGAGTATGGGAGAGGATGGTAGAATTCCAGGTGTAGCGGTGAAATGCGTAGAGATC TGGAGGAATACCGATGGCGAAGGCAGCCATCTGGGCCTAATACTGACACTGAGGTACG AAAGCATGGGGAGCAAACAGGATTAGATACCCTGGTAGTCCATGCCGTAAACGATGTC TACTAGCCGTTGGGGTCTTTGAGACTTTAGTGGCGCAGCTAACGCGATAAGTAGACCG CCTGGGGAGTACGGTCGCAAGACTAAAACTCAAATGAATTGACGGGGGCCCGCACAA GCGGTGGAGCATGTGGTTTAATTCGATGCAACGCGAAGAACCTTACCTGGTCTTGACAT AGTAAGAACTTTCCAGAGATGGATT GGTGCCTTCGGGAGCTTACATACAGGTGCTGCAT GGCTGTCGTCAGCTCGTGTCGTGAGATGTTGGGTTAAGTCCCGCAACGAGCGCAACCC TTTTCCTTATTTGCCAGCGGGTTAAGCCGGGAACTTTAAGGATACTGCCAGTGACAAAC TGGAGGAAGGCGGGGACGACGTCAAGTCATCATGGCCCTTACGACCAGGGCTACACA CGTGCTACAATGGTCGGTACAAAGGGTTGCTACCTAGCGATAGGATGCTAATCTCAAAA AGCCGATCGTAGTCCGGATTGGAGTCTGCAACTCGACTCCATGAAGTCGGAATCGCTA GTAATCGCGGATCAGAATGCCGCGGTGAATACGTTCCCGGGCCTTGTGCACACCGCCC GTCACACCATGGGAGTTTGTTGCACCAGTAGTAGGTAGTCTAACCGCAAGGAGGACGC TTACCACGGTGTGGCCGATGACTGGGGTGAAGTCGTAACAAGGTAACCGTA

Fig. 2. A) PCR amplification of $16 \mathrm{~S}$ rDNA gel electrophoresis of strain CY1. Line 1 is DNA marker, line 2 is $16 \mathrm{~S}$ rDNA. B) $16 \mathrm{~S}$ rDNA fragment size is $1505 \mathrm{bp}$, and the primers are the red sequences.

$16 S$ rDNA gene sequences analysis. The 16S rDNA gene sequences of the isolate CY1 was approximately $1500 \mathrm{bp}$ in length (Fig. 2A), and demonstrated a restriction fragment pattern identical to that of DNA extracted from the original $A$. Iwoffii ATCC17925. Figure 2B shows the PCR products of the expected 1505 bp $16 \mathrm{~S}$ rDNA fragment size of the CY1 strain. The results of Blast alignments showed that the isolate CY1 shared the highest $16 \mathrm{~S}$ rDNA sequence identities $(98.08 \%)$ with $A$. Lwoffii type 
strain ATCC17925 (HE651921). The phylogenetic tree based on 16S rDNA sequences showed that the isolate CY1 is most closely related to the new strains A. Iwoffii ATCC 17925 (Fig.3).

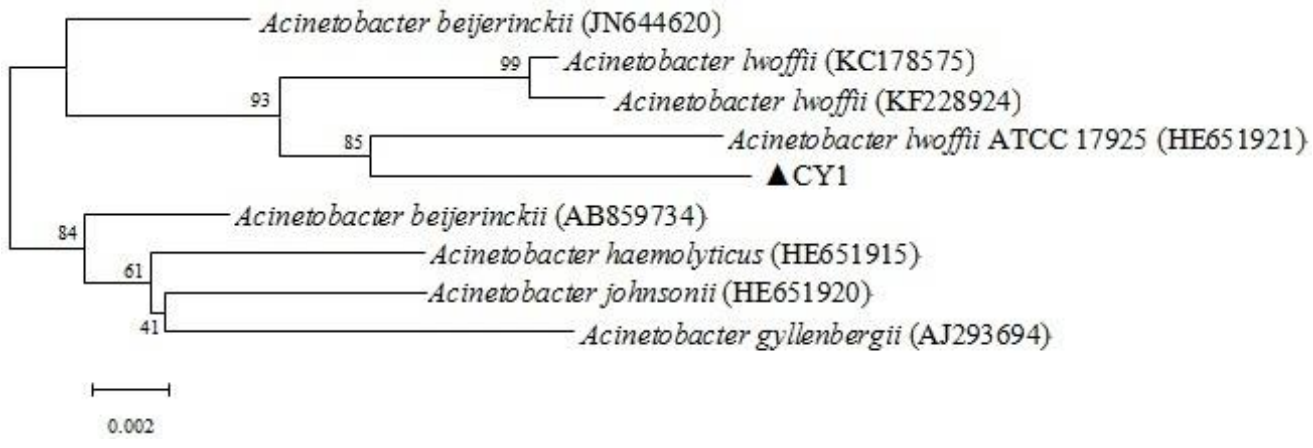

Fig. 3. Phylogenetic tree analysis of Acinetobacter species based on $16 S$ rDNA nucleotide sequences. Unrooted tree was generated using neighbor-joining method by the MEGA4.1 software. The numbers next to the branches indicate percentage values for 1000 bootstrap replicates. The scale bar represents 0.002 substitutions per site. Bootstrap values above $45 \%$ are shown at the nodes. GenBank accession numbers of type strains are given in brackets, and the isolate CY1 identified in this study is indicated by the shaded triangle.

Experimental infection results. Following the infection with strain $\mathrm{CY} 1$, mortality rate was $75 \%$. The dead grass carp exhibited hemorrhages on the pectoral, ventral, and anal fins, and on their bodies.

Antimicrobial susceptibility test. The results of antimicrobial susceptibility tests showed that the isolate CY1 strain had high susceptibility to most of the tested antimicrobial agents, including aminoglycosides, $\beta$-lactams (penicillins and cephalosporins), quinolones (except norfloxacin) and cephalosporin (except cefixime); intermediate susceptibility to sulphonamides, cefixime, norfloxacin and erythrocin; low susceptibility to clindamycin, vancomycin, teicoplanin and trimethoprim (Table 2).

Table 2. Antimicrobial sensitivity test of $\mathrm{CY} 1$ strain

\begin{tabular}{|c|c|c|c|c|c|}
\hline Antimicrobial & $\begin{array}{c}\text { Concentration } \\
(\mu \mathrm{g} / \text { piece })\end{array}$ & $\begin{array}{c}\text { inhibition zone } \\
\text { diameter } \\
\text { CY1 }\end{array}$ & Antimicrobial & $\begin{array}{l}\text { Concentr } \\
\text { ation }(\mu g \\
\text { /piece) }\end{array}$ & $\begin{array}{c}\text { inhibition zone } \\
\text { diameter } \\
\text { CY1 }\end{array}$ \\
\hline Amoxicillin & 10 & $28 \mathrm{H}$ & Gentamicin & 10 & $25 \mathrm{H}$ \\
\hline Piperacillin & 100 & $23 \mathrm{H}$ & Kanamycin & 30 & $28 \mathrm{H}$ \\
\hline Ampicillin & 10 & $29 \mathrm{H}$ & Netilmicin & 30 & $30 \mathrm{H}$ \\
\hline Cefoperazone & 75 & $17 \mathrm{H}$ & Sulfafurazole & 300 & $14 M$ \\
\hline Cefotaxime & 30 & $20 \mathrm{H}$ & Cotrimoxazole & $\begin{array}{c}23.75 / 1 \\
25\end{array}$ & $18 \mathrm{H}$ \\
\hline $\begin{array}{l}\text { Cefalotin } \\
\text { Cefamandole } \\
\text { Cefixime }\end{array}$ & $\begin{array}{c}30 \\
30 \\
5\end{array}$ & $\begin{array}{l}7 \mathrm{~N} \\
18 \mathrm{H} \\
14 \mathrm{M}\end{array}$ & $\begin{array}{l}\text { Trimethoprim } \\
\text { Vancomycin } \\
\text { Teicoplanin }\end{array}$ & $\begin{array}{c}5 \\
30 \\
30\end{array}$ & $\begin{array}{l}N \\
7 N \\
N\end{array}$ \\
\hline CephalosporinIV & 30 & $18 \mathrm{H}$ & Tetracycline & 30 & $30 \mathrm{H}$ \\
\hline Erythrocin & 15 & $12 M$ & Chloromycetin & 30 & $36 \mathrm{H}$ \\
\hline Azithromycin & 15 & $9 N$ & Nitrofurantoin & 300 & $14 M$ \\
\hline Norfloxacin & 10 & $11 M$ & Rifampicin & 5 & $23 \mathrm{H}$ \\
\hline Nalidixic acid & 30 & $24 \mathrm{H}$ & Metronidazole & 5 & N \\
\hline Ofloxacin & 5 & $24 \mathrm{H}$ & Nystain & 100 & $\mathrm{~N}$ \\
\hline Clidamycin & 2 & $\mathrm{~N}$ & Enrofloxacin & 5 & $36 \mathrm{H}$ \\
\hline Neomycin & 30 & $32 \mathrm{H}$ & Levofloxacin & 5 & $36 \mathrm{H}$ \\
\hline Imipenem & 10 & $37 \mathrm{H}$ & Meropenem & 10 & $35 \mathrm{H}$ \\
\hline Streptomycin & 10 & $31 \mathrm{H}$ & Enoxacin & 10 & $30 \mathrm{H}$ \\
\hline Pefloxacin & 10 & $35 \mathrm{H}$ & & & \\
\hline
\end{tabular}

$\mathrm{H}$ : inhibition zone diameter $>15 \mathrm{~mm}$. $\mathrm{M}: 10 \mathrm{~mm} \leqq$ inhibition zone diameter $\leqq 14 \mathrm{~mm} . \mathrm{N}$ : inhibition zone diameter $<10 \mathrm{~mm}$ 


\section{Discussion}

Numerous published studies focusing on the diversity of Acinetobacter spp. refer to the potential of some members of this genus to act as opportunistic pathogens in higher vertebrates, and development of antibiotic resistance (Ku et al., 2000; Joly-Guillou et al., 2005). Acinetobacter baumannii, Acinetobacter johnsonii, and A. Iwoffii, have emerged as important nosocomial pathogens because of their persistence in the hospital environment and broad antimicrobial drug resistance patterns (Bergogne-Berezin et al., 1996; Murray et al., 2005). They are often associated with clinical illness including bacteremia, pneumonia, meningitis, peritonitis, endocarditis, and infections of the urinary tract and skin (Rathinavelu al et al., 2003; Takehiko et al., 2005; Ku et al., 2000). One of the main reasons for disease caused by Acinetobacter is, in part, due to their capability to form biofilms (Idzenga et al., 2006; Medina et al., 2007; Henwood et al., 2002). Another reason is the fact that clinical isolates of these bacteria are frequently resistant to the major antibiotics used to treat nosocomial infection (Henwood et al., 2002; Murray et al., 2005; Srinivasan et al., 2009; Towner et al., 1997; Zhang et al., 2013).

Although Acinetobacter spp. can also cause infections in freshwater fish, to our knowledge, the diversity and dynamics of members of this genus in grass carp has not been addressed in literature (Towner et al., 2006; Carlos et al., 2013; Dadar et al., 2016). Some studies only characterize the bacterial community; they do not report the lethality of Acinetobacter spp. in freshwater fish (Gonzalez et al., 2000). In this study, cultivable $A$. Iwoffii resulted in a mortality rate of $75 \%$ at a dose of $3.83 \times 10^{8} \mathrm{CFU} / \mathrm{mL}$ and was confirmed as a pathogen of grass carp with a severe mortality rate. In this study, $A$. Iwoffii strain CY1 showed low susceptibility to clidamycin, trimethoprim, and teicoplanin. However, it showed high susceptibility to aminoglycosides, $\beta$-lactams, and quinolones, which provides information for clinical treatment and infection prevention caused by $A$. Iwoffii in fish. This new finding in fish is of concern, as the use of antibiotics may disrupt the normal flora and facilitate the emergence of opportunistic infection. Understanding Acinetobacter virulence factors is still at an elementary stage. However, the ability of Acinetobacter spp. to produce extracellular enzymes and toxins, their ability of adherence to epithelial cells, the role of polysaccharidic capsule, and surface components protecting the bacteria from phagocytosis has been reported (Srinivasan et al., 2009; Grziela et al., 2008). The study of Acinetobacter virulence factors and correct adaptation of the use of antibiotics capable of eradicating them is imperative.

In summary, this is the first report of $A$. Iwoffii from grass carp. Experimental challenges were conducted and demonstrated the pathogenicity of the A. Iwoffii isolate for grass carp. 16S rDNA gene sequence analysis of isolate CY1 will provide scientific reference for fish disease diagnostics. Further research is needed to investigate the virulence of $A$. Iwoffii isolated from farmed fish.

\section{Acknowledgements}

This study was supported by the National Natural Science Foundation of China (No. 31272692), Key Scientific Research Project Universities and Colleges in Henan province (No. 17A240001), Natural Science Foundation of Tianjin city (No. 16JCZDJC33500, 15JCZDJC34000), Innovation Team of Tianjin Fisheries Research System (No. ITTFRS2017009) and Team of Provincial Science and Technology Innovation of Henan High Education (No. 15IRTSTHN018).

\section{References}

Bergogne-Berezin E., K.J. Towner, 1996. Acinetobacter spp. as nosocomial pathogens: microbiological, clinical, and epidemiological features. Clin Microbiol Rev, 9:148-165.

Carlos N.R, I.V. Moreira, L.S. Stadler, R.B. Moore and C.M. Manaia, 2013. Diversity and antibiotic resistance of Acinetobacter spp.in water from the source to the tap. Appl Microbiol Biotechnol, 97:329-340.

Dadar M., M. Adel and M J. Zorriehzahra, 2016. Isolation and phylogenic analysis of emerging new antibiotic resistant bacteria, Acinetobacter Iwoffii, associated with mortality in farmed rainbow trout. Iranian J Fish Sci, 15(4):1279-1292. 
Forster D.H., F.D. Daschner, 1998. Acinetobacter species as nosocomial pathogens. Eur J Clin Microbiol Infect Dis, 17:73-77.

Gonzalez C.J., J.A. Santos, M.L. Garcia-Lopez and A. Otero, 2000. Psychrobacters and related bacteria in freshwater fish. J Food Prot, 63:315-321.

Grziela B., 2008. Virulence Mechanisms of Acinetobacter. Springer. In book: Acinetobacter Biology and Pathogenesis, pp.145-154.

Henwood C.J., T. Gatward, M. Warner, D. James, M.W. Stockdale, R.P. Spence, K.J. Towner, D.M. Livermore and N. Woodford, 2002. Antibiotic resistance among clinical isolates of Acinetobacter in the UK, and in vitro evaluation of tigecycline (gar936). J Antimicrob Chemother, 49:479-487.

Hu X., H. Li and A. Lü, 2010. Isolation of bacteria from intestine in Carp (Cyprinus Carpio) and their physiological and biochemical characteristics. Chinese Agricultural Science Bulletin. 26(14):365-367.

Idzenga D., M.A. Schouten and A.R. van Zanten, 2006. Outbreak of Acinetobacter genomic species 3 in a Dutch intensive care unit. J Hosp Infect, 63:485-487.

Joly-Guillou M.L., 2005. Clinical impact and pathogenicity of Acinetobacter. Clin. Microbiol. Infect, 11:868-873.

Kim M.G., J.W. Jeon and I.H. Ryu, 2014. Mycotic Abdominal Aortic Aneurysm Caused by Bacteroides Thetaiotaomicron and Acinetobacter Lwoffii: The First Case in Korea. Infect Chemother, 46(1):54-58.

Kozińska A., E. Paździor, A. Pękala and W. Niemczuk, 2014. Acinetobacter johnsonii and Acinetobacter Iwoffii-the emerging fish pathogens. Bulletin of the Veterinary Institute in Pulawy, 58(2):193-199.

Ku, S.C., P.R. Hsueh, P.C. Yang and K.T. Luh, 2000. Clinical and Microbiological Characteristics of Bacteremia Caused by Acinetobacter Iwoffii. Eur J Clin Microbiol Infect Dis, 19:501-505.

Luciano T., R. Katia and O. Donatella, 2007. Catheter related bacteremia and multidrug resistant Acinetobacter Iwoffii. Emerg Infect Dis, 13:355-356.

Lü A., Y. Song, X. Hu, J. Sun, L. Li, C. Pei, C. Zhang and G. Nie, 2016. Aeromonas veronii, Associated with skin ulcerative syndrome, isolated from the Goldfish (Carassius auratus) in China. The Israeli Journal of Aquaculture-Bamidgeh, 68:1-10.

Medina J., C. Formento, J. Pontet, A. Curbelo, C. Bazet, J. Gerez and E. Larranaga, 2007. Prospective study of risk factors for ventilator-associated pneumonia caused by Acinetobacter species. J Crit Care, 22:18-26.

Murray C.K., D.R Hospenthal, 2005. Treatment of multidrug resistant Acinetobacter. Curr Opin Infect Dis, 18:502-506.

Rathinavelu S., Y. Zavros and J.L. Merchant, 2003. Acinetobacter Iwoffii infection and gastritis. Microbes Infect, 5:651-657.

Sule O., H.A. Ludlam, C.W. Walker, D.F. Brown and M.E. Kaufmann A, 1997. pseudo outbreak of respiratory infection with Acinetobacter species. InfectControl Hosp Epidemiol, 18:510-512.

Srinivasan V.B., G. Rajamohan and P. Pancholi, 2009. Genetic ralateness and molecular characterization of multidrug resistant Acinetobacter baumannii isolated in central Ohio, USA. Ann Clin Microbiol Antimicrob, 8:21.

Takehiko M., T. Nakazato, R. Yamazaki, Y. Ikeda and S. Okamoto, 2005. Acinetobacter Iwoffii Septicemia Associated with a Peripheral Intravascular Catheter. Internal medicine, 45:1580.

Towner K.J., 1997. Clinical importance and antibiotic resistance of Acinetobacter spp. J Med Microbiol,46:721-746.

Towner K., 2006. The Genus Acinetobacter. Prokaryotes, 6:746-758.

Toyoshima M.I., K.I. Chida and A.A. Suda, 2010. Fulminant community-acquired pneumonia probably caused by Acinetobacter Iwoffii. Respirology, 15:860-868.

Wareham D.W., D.C. Bean and P. Khanna, 2008. Bloodstream infection due to Acinetobacter spp: epidemiology, risk factors and impact of multi-drug resistance. Eur J Clin Microbiol Infect Dis, 27:607-12.

Zhang W., H. Liang, L. Liu, G. Peng and H. Hu, 2013. Identification on Multidrug resistance Gene of New Delhi metall-beta-lactamase-1 in Acinetobacter Iwoffii. Med J Chin PAPF, 24(4):13-16. 